\title{
Perfluoroctano em cavidade orbitária após vitrectomia posterior e sutura de perfurante ocular: relato de caso
}

\author{
Perfluoro-n-octane in orbital cavity after posterior vitrectomy and suture of eye \\ perforating injury: case report
}

Marcelo Mendes Lavezzo ${ }^{1}$, Kenzo Hokazono ${ }^{2}$, Leandro Cabral Zacharias ${ }^{2}$, Walter Yukihiko Takahashi ${ }^{2}$

\section{RESUMO}

O objetivo é relatar, pela primeira vez, o caso de um paciente submetido à vitrectomia posterior e sutura de perfurante ocular que evoluiu com extravasamento de perfluoroctano para cavidade orbitária. Paciente do sexo masculino, 39 anos, hígido, encaminhado para avaliação oftalmológica após ter sofrido trauma no olho direito com pedaço de metal há um dia. Ao exame, apresentava redução súbita da acuidade visual à direita com ferimento perfurante ocular córneo-escleral, corpo estranho intraocular e descolamento de retina. O paciente foi submetido à sutura de perfurante, introflexão escleral e vitrectomia posterior com utilização de perfluoroctano, quando foi constatada transfixação do globo ocular. No pós-operatório, evoluiu com proptose e presença de imagens hiperdensas à tomografia computadorizada de órbitas, sugerindo tratar-se de extravasamento de perfluoroctano para a cavidade orbitária. Dessa forma, diante de ferimento perfurante ocular, deve--se sempre atentar para a possibilidade de existência de corpo estranho intraocular, bem como para possível ocorrência de transfixação do globo ocular, no momento de se realizar a programação cirúrgica destes casos.

Descritores: Ferimentos oculares penetrantes; Corpos estranhos no olho; Vitrectomia; Fluorcarbonetos; Humanos; Masculino; Adulto; Relato de caso

\begin{abstract}
A case of a perfluoro-n-octane leakage into the orbital cavity after corneoscleral suture, scleral buckling and pars plana vitrectomy in an eye with perforating injury after trauma is reported for the first time. A previously healthy 39-year-old man wassent for ophthalmic evaluation one day after suffering a penetrating ocular trauma in his right eye while hammering a nail. On the initial evaluation, the patient presented sudden reduction of visual acuity on his right eye with a perforating corneoscleral injury, intraocular foreign body and retinal detachment. The patient was submitted to corneoscleral suture, pars plana vitrectomy with perfluoro-n-octane administration to flatten the retina and scleral buckling, when it was found transfixation of the globe by the intraocular foreign body. Postoperatively, computed tomography scans of the orbit were ordered due to proptosis, which showed the presence of hyperdense images, suggesting leakage of perfluoro-n-octane into the orbital cavity. Thus, in cases of perforating eye injury, one should be suspicious about the possibility of intraocular foreign body, as well as possible occurrence of transfixation of the globe when scheduling the surgery.
\end{abstract}

Keywords: Eye injuries, penetrating; Eye foreign bodies; Vitrectomy; Fluorocarbons, Humans; Male; Adult; Case report

\section{INTRODUÇÃO}

O perfluoroctano (PFO) é um perfluorocarbono líquido usado como auxílio para o reparo cirúrgico da vitreorretinopatia proliferativa e de roturas retinianas gigantes ${ }^{(1)}$. Sua alta densidade, baixa viscosidade e clareza óptica permitem uma força de tamponamento que estabiliza a retina e permite sua manipulação com maior facilidade (2).

O PFO é removido no intraoperatório por seu alto risco de toxicidade retiniana ${ }^{(3)}$. Entretanto, uma pequena quantidade de PFO é eventualmente deixada inadvertidamente na cavidade vítrea ao final da cirurgia, ocorrendo em 7,8 - 38,3\% dos casos, dependendo do tipo utilizado ${ }^{(4,5)}$.

Devido à sua alta densidade, o PFO pode aparecer radiopaco e, por conseguinte, mimetizar um corpo estranho intraocular (CEIO) ou coleção hemorrágica. À medida que o uso do PFO tem se tornado cada vez mais comum na cirurgia retiniana, o seu reconhecimento em exames de imagem tornou-se importante ${ }^{(6)}$.

Neste relato, é descrito um paciente apresentando ferimento perfurante ocular córneo-escleral, CEIO e descolamento de retina, que durante o tratamento cirúrgico evoluiu com extravasamento de PFO para cavidade orbitária, através de lesão posterior que caracterizou transfixação do bulbo ocular. Não foi encontrado nenhum outro relato semelhante na literatura.

\section{RELATO DE CASO}

Paciente, 39 anos, masculino, branco, hígido, encaminhado ao Pronto Socorro de Oftalmologia por história de um dia de trauma ocular à direita com pedaço de metal, enquanto martelava um prego, evoluindo com redução súbita da acuidade visual (AV) do olho direito (OD). Negava alterações no olho esquerdo (OE).

Ao exame, apresentava AV de movimento de mãos (MM) no OD e de 20/20 no OE. À biomicroscopia do OD apresentava: ferimento perfurante córneo-escleral, câmara anterior formada, hifema $(1,0 \mathrm{~mm})$ ausência de Seidel e cristalino transparente. Biomicroscopia e fundoscopia do OE foram normais e a pressão intraocular (PIO) era de $16 \mathrm{mmHg}$. À fundoscopia do OD, foi observada hemorragia vítrea.

Procedeu-se à realização de radiografia de crânio, sendo constatada imagem sugestiva de CEIO (Figuras 1A e 1B). A ultrassono-
Submitted for publication: December 19, 2009

Accepted for publication: November 29, 2010

Study carried out at the Clínica Oftalmológica do Hospital das Clínicas da Faculdade de Medicina da Universidade de São Paulo - USP - São Paulo (SP), Brazil.

${ }^{1}$ Physician, Clínica Oftalmológica, Hospital das Clínicas, Faculdade de Medicina, Universidade de São Paulo - USP - São Paulo (SP), Brazil.

2 Physician, Setor de Retina e Vítreo, Clínica Oftalmológica, Hospital das Clínicas, Faculdade de Medicina, Universidade de São Paulo - USP - São Paulo (SP), Brazil.
Funding: No specific financial support was available for this study.

Disclosure of potential conflicts of interest: M.M.Lavezzo, None; K. Hokazono, None; L.C.Zacharias, None; W.T.Takahashi; None.

Correspondence address: Marcelo Mendes Lavezzo. Rua Capote Valente, 640 - Ap. 23 - São Paulo (SP) - 05409-002 - Brazil - E-mail: mmlavezzo@yahoo.com.br 
grafia do OD evidenciou presença de opacidades vítreas difusas e membranáceas no vítreo, descolamento de retina no setor superior e suspeita de CEIO (Figuras 1C e 1D).

O paciente foi submetido à sutura de perfurante ocular, introflexão escleral (implante superior $180^{\circ}$ e faixa $360^{\circ}$ ) e WPP 20-gauge, com manutenção da pressão de infusão constante entre $35-40 \mathrm{~cm}$ de altura da garrafa acima da altura do paciente, injeção de PFO (durante a qual foi fechada a infusão) e aplicação de endolaser ao redor de rotura gigante próxima à arcada temporal superior. Neste local, foi visualizado o CEIO e procedeu-se à sua retirada. Pôde-se observar esclera nesta localização, demonstrando que houvera transfixação do globo ocular. Após isso, aplicou-se endolaser e realizou-se inspeção da base vítrea, sendo visualizada pequena rotura em periferia nasal superior, que foi prontamente bloqueada com o endolaser. Procedeu-se à retirada do PFO, troca fluido-gasosa e injeção de óleo de silicone.

No quarto dia de pós-operatório, apresentava AV de MM a um metro, evoluindo com edema palpebral e proptose. Não apresentou hipertermia ou outros sinais flogísticos sugestivos de processo infeccioso, como celulite orbitária. Diante disto, optou-se pela reali-
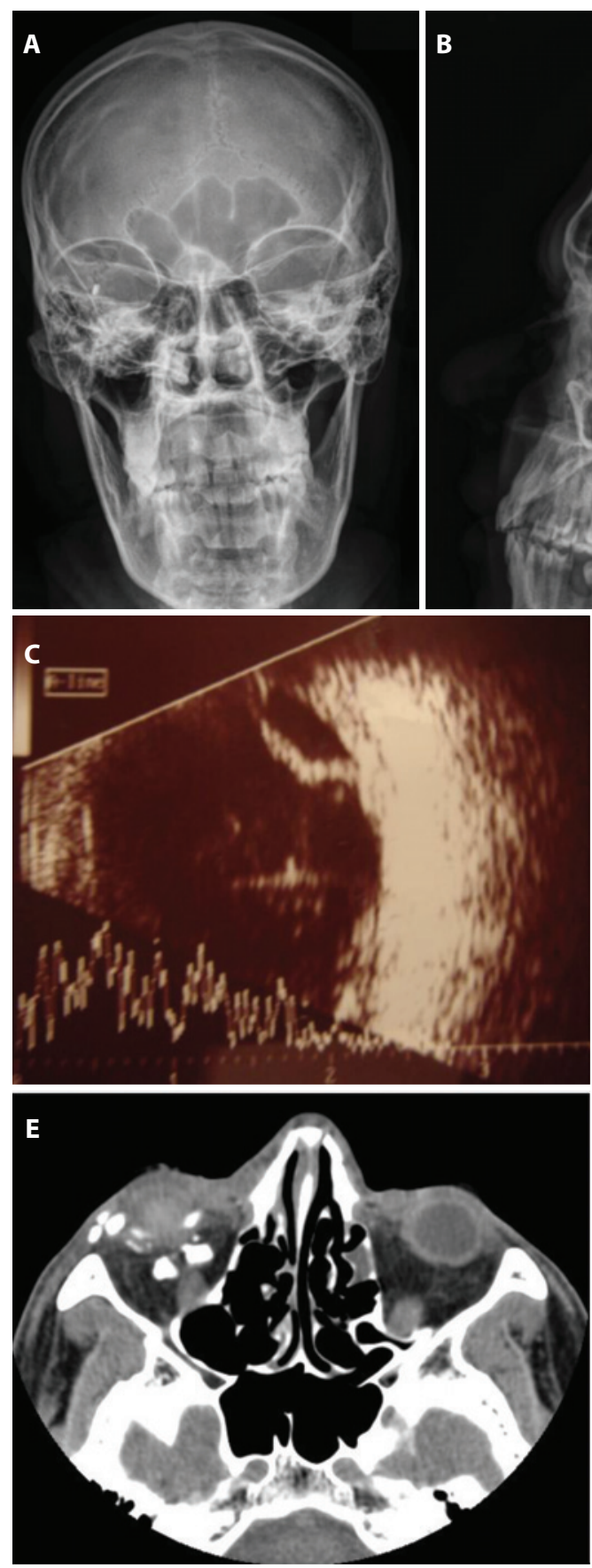

Figura 1. Radiografias de crânio $(A$ e B revelando imagem sugestiva de corpo estranho intraocular à direita. Ultrassonografia do olho direito apresentando: (C) Descolamento de retina no setor superior; (D) Suspeita de CEIO no vítreo. Tomografia computadorizada de órbitas (EeF) evidenciando inúmeras imagens hiperdensas em regiâo periocular e da cavidade orbitária, sugestivas de perfluoroctano.
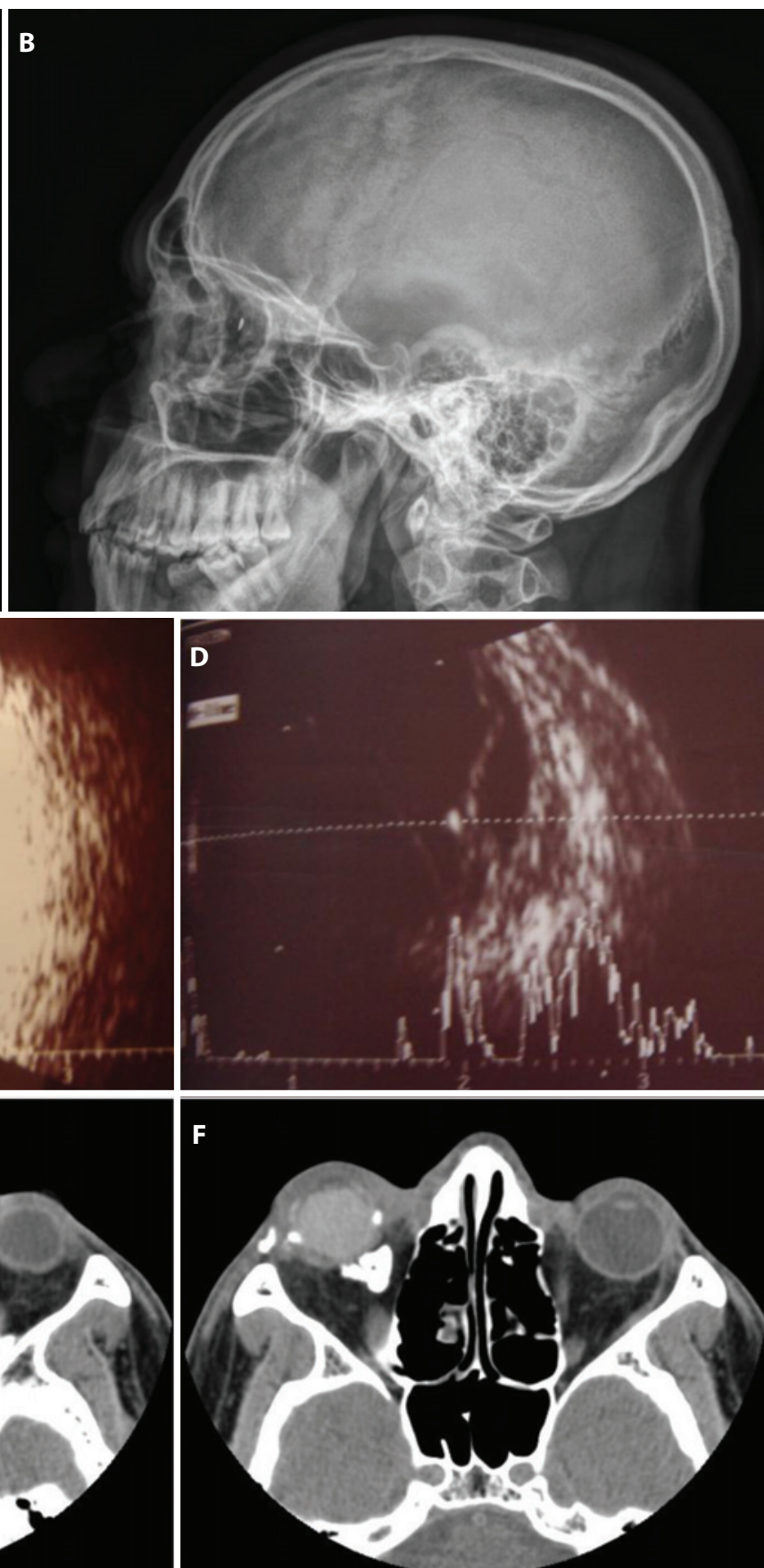
zação da tomografia computadorizada (TC) de órbitas, que evidenciou inúmeras imagens hiperdensas na região periocular e na cavidade orbitária (Figuras 1E e 1F), cujo aspecto era sugestivo de PFO, considerando o histórico do paciente e o procedimento cirúrgico ao qual fora submetido.

No sétimo dia de pós-operatório, apresentava AV de conta dedos a um metro, hemorragia pré-retiniana, retina aplicada e marcas de laser próximo à arcada temporal superior (Figuras 2A-E).

O paciente mantém seguimento ambulatorial apresentando retina aplicada até o momento. Quanto à proptose, durante um ano de seguimento, o paciente não apresentou dor ou outras queixas, sendo que houve estabilização do quadro, optando-se pelo acompanhamento clínico (Figura 2F). Não existem medidas exoftalmométricas prévias à cirurgia, porém a medida atual do paciente, utilizando-se o exoftalmômetro de Hertel, é de $18 \mathrm{~mm}$ no OD e $14 \mathrm{~mm}$ no OE, sendo $124 \mathrm{~mm}$ a base. Tal medida se manteve durante um ano de seguimento, ficando difícil a estimação do volume orbitário de PFO.

\section{DISCUSSÃO}

O PFO $\left(\mathrm{C}_{8} \mathrm{~F}_{18}\right)$ é um composto sintético do carbono totalmente fluorado, de uso muito difundido em procedimentos cirúrgicos retinianos. Apresenta alta densidade e baixa viscosidade ${ }^{(7)}$, promovendo uma força de tamponamento que auxilia a estabilizar a retina no intraoperatório e permitindo outras manobras como extrusão de fluido subretiniano, manipulação das bordas retinianas e aplicação de laser ${ }^{(6)}$. Também possui interface mais visível, o que auxilia na sua completa remoção no intraoperatório, apresenta menor viscosidade oferecendo menos resistência para injeção/aspiração através dos instrumentos microcirúrgicos e possui pressão de vapor mais alta, permitindo evaporação mais completa após a troca fluido-gasosa ${ }^{(8)}$.

Alguns autores estudaram o efeito clínico-patológico do PFO retido no interior de olhos de coelhos. O exame histopatológico demonstrou macrófagos e células gigantes multinucleadas ${ }^{(2)}$. O PFO retido por até 48 horas não causou evidência de toxicidade. Toda-
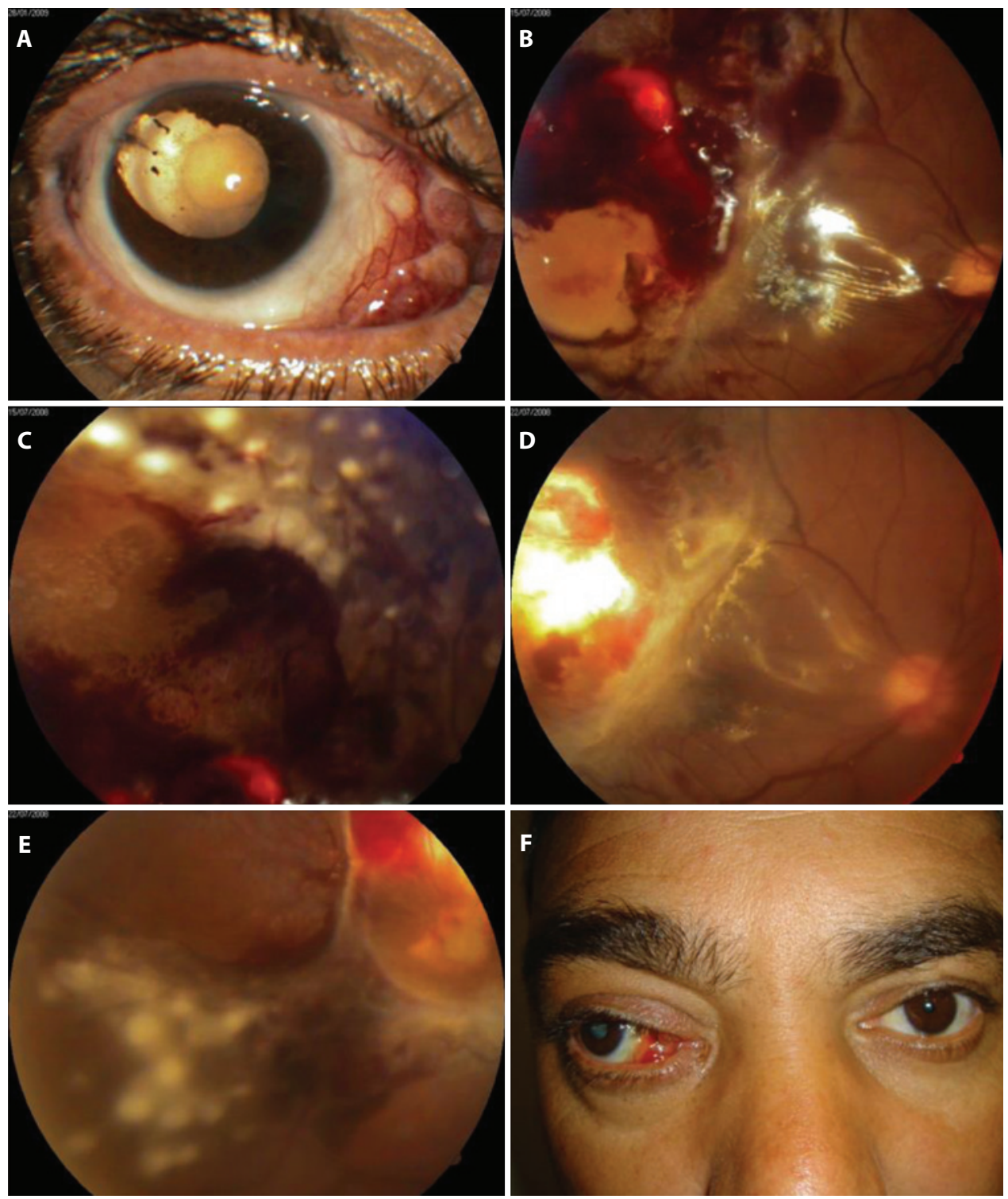

Figura 2. Quarto dia pós-operatório: (A) Corectopia e granulomas nas regiões das esclerotomias; (B) Hemorragias pré-retinianas no polo posterior; (C) Marcas de laser. Vigésimo dia pós-operatório: (D) e (E) Aspecto do polo posterior apresentando retina aplicada e marcas de laser próximo à arcada temporal superior; (F) Proptose à direita. 
via, pequenas quantidades de PFO $(0,1 \mathrm{ml})$ retidas por mais tempo levaram à deposição de precipitados brancos, com acúmulo de células na interface do PFO e vítreo cortical comprimido. A evidência de formação progressiva de membrana epirretiniana também foi notada ${ }^{(2)}$.

Os precipitados observados nos olhos eram mais aderentes na cápsula posterior e retina periférica. Essa resposta parece ser mais exacerbada em indivíduos jovens, olhos com considerável vítreo residual e retenção de $\mathrm{PFO}$ superior a 0,25 m/(2). Caso haja retenção de PFO, recomenda-se que olhos que desenvolvam inflamação persistente, formação de membrana secundária ou descolamento de retina recorrente devem ser submetidos à reoperação para remoção deste material (2).

No caso de PFO subfoveal retido, haveria danos aos fotorreceptores e alterações retinianas irreversíveis (inflamação e atrofia retinianas ${ }^{(3)}$, degeneração retiniana e toxicidade ao epitélio pigmentar da retina $\left.a^{(9)}\right)$.

Não há dados na literatura que descrevem o que poderia acontecer na órbita do paciente em questão e, devido à raridade deste acontecimento, é difícil prever o resultado a longo prazo. Mas, até o presente momento, não há indícios de inflamação orbitária e a proptose se mantém estável, sem prejuízos secundários à córnea ou compressão perceptível de estruturas orbitárias.

Alguns autores fizeram um experimento com olho porcino enucleado, injetando PFO em seu interior. O seu aspecto, à tomografia computadorizada (TC), mostrou o PFO como uma coleção fluida globular suavemente marginada, redonda e atenuada na porção posterior da cavidade vítrea ${ }^{(6)}$. Imagens semelhantes puderam ser observadas no caso relatado.

As imagens hiperdensas na região periocular e cavidade orbitária observadas na TC foram sugestivas de PFO, considerando-se a literatura já apresentada e o histórico do paciente, fator fundamental ao se definir um diagnóstico diferencial.
O presente trabalho apresenta limitações, principalmente devido ao seguimento por período curto de tempo e à limitação a apenas um paciente. Assim, fica difícil realizar quaisquer recomendações de como proceder diante destes casos. Entretanto, no paciente em questão, nota-se que os resíduos de PFO foram bem tolerados pelos tecidos adjacentes, não havendo indícios de inflamação orbitária ou proptose progressiva. Estudos em cobaias poderiam ser realizados com o intuito de verificar possível ação inflamatória do PFO em tecidos orbitários, e mais relatos ou séries de casos semelhantes ajudariam a compreender melhor tal complicação. Ademais, diante de ferimento perfurante ocular, deve-se sempre atentar para a possibilidade de existência de CEIO, bem como para possível ocorrência de transfixação do globo ocular, no momento de se realizar a programação cirúrgica destes casos.

\section{REFERÊNCIAS}

1. Chang S. Low viscosity liquid fluorochemicals in vitreous surgery. Am J Ophthalmol. 1987;103(1):38-43

2. Elsing SH, Fekrat S, Green WR, Chang S, Wajer SD, Haller JA. Clinicopathologic findings in eyes with retained perfluoro-n-octane liquid. Ophthalmology. 2001;108(1):45-8.

3. Chang S, Sparrow JR, Iwamoto T, Gershbein A, Ross R, Ortiz R. Experimental studies of tolerance to intravitreal perfluoro-n-octane liquid. Retina. 1991;11(4):367-74.

4. Bourke RD, Simpson RN, Cooling RJ, Sparrow JR. The stability of perfluoro-N-octane during vitreoretinal procedures. Arch Ophthalmol. 1996;114(5):537-44.

5. Scott IU, Murray TG, Flynn HW Jr, Smiddy WE, Feuer WJ, Schiffman JC. Outcomes and complications associated with perfluoro-n-octane and perfluoroperhydrophenanthrene in complex retinal detachment repair. Ophthalmology. 2000;107(5):860-5.

6. Christoforidis JB, Caruso PA, Curtin HD, Fiore T, D'Amico DJ. CT characteristics of intraocular perfluoro-N-octane. AJNR Am J Neuroradiol. 2003;24(9):1769-71.

7. Ryan SJ. Retina. Philadelphia: Mosby; 2001.

8. Chang S. Perfluorocarbon liquids in vitreoretinal surgery. Int Ophthalmol Clin. 1992;32(2): 153-63.

9. Sakurai E, Ogura Y. Removal of residual subfoveal perfluoro-n-octane liquid. Graefes Arch Clin Exp Ophthalmol. 2007;245(7):1063-4

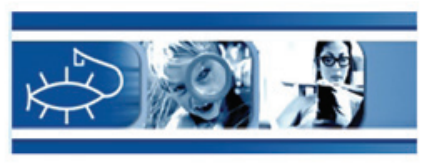

\title{
Envie seu trabalho pelo site do ABO
}

\author{
www.scielo.br/abo
}

